\title{
Sex- and Disease-Specific Inflammasome Signatures in Circulating Blood Leukocytes of Patients with Abdominal Aortic Aneurysm
}

\author{
Xiaoyu Wu, ${ }^{1,2}$ Sinan Cakmak, ${ }^{2}$ Markus Wortmann, ${ }^{2}$ Maani Hakimi, ${ }^{2,3}$ Jian Zhang, ${ }^{1}$ Dittmar Böckler, ${ }^{2}$ \\ and Susanne Dihlmann ${ }^{2 *}$
}

${ }^{1}$ Department of Vascular \& Thyroid Surgery, The First Hospital of China Medical University, Shenyang, China; ${ }^{2}$ University Hospital Heidelberg, Department of Vascular and Endovascular Surgery, Heidelberg, Germany; and ${ }^{3}$ Vaskuläre Biomaterialbank Heidelberg (VBBH), Heidelberg, Germany

\begin{abstract}
Male sex is a risk factor for abdominal aortic aneurysm (AAA). Within the AAA adventitia, infiltrating leukocytes express high levels of inflammasome components. To further elucidate the role of inflammatory cells in the pathogenesis of AAA, we here addressed expression and functionality of inflammasome components in peripheral blood mononuclear cells (PBMC) of AAA patients in association with sex. PBMC and plasma were isolated from 100 vascular patients, including 34 pairs of AAA patients and age/sexmatched non-AAA patients. Male PBMC were found to express significantly higher mRNA levels of AIM2, NLRP3, ASC (PYCARD), CASP1, CASP5, and ILIB (all $P<0.0001$ ) than female PBMC. Within the male patients, PBMC of AAA patients displayed increased mRNA levels of NLRP3 $(P=0.044), C A S P 1(P=0.032)$ and $I L I B(P=0.0004)$ compared with matched non-AAA PBMC, whereas there was no difference between female AAA and non-AAA patients. The relative protein level of NLRP3 was significantly lower in PBMC lysates from all AAA patients than in matched controls $(P=0.038)$, whereas AIM2 and active Caspase-1 (p10) protein levels were significantly increased $(P=0.014$ and $P=0.049$ ). ELISA revealed significantly increased IL- $1 \alpha(\mathrm{mean}=6.34 \mathrm{versus} 0.01 \mathrm{pg} / \mathrm{mL})$ and IL-1 $\beta$ plasma levels (mean $=12.07$ versus $0.04 \mathrm{pg} / \mathrm{mL}$ ) in AAA patients. The data indicate that male PBMC display a systemic proinflammatory state with primed inflammasomes that may contribute to AAA-pathogenesis. The AAA-specific inflammasome activation pattern suggests differential regulation of the sensors AIM2 and NLRP3 in inflammatory cells of AAA patients.
\end{abstract}

Online address: http://www.molmed.org

doi: 10.2119/molmed.2016.00035

\section{INTRODUCTION}

Dilatation and potential rupture of abdominal aortic aneurysm (AAA) result in high morbidity and mortality rates. Epidemiological studies identified age, hypertension, smoking and male sex as major risk factors for AAA. Currently, surgical or endovascular repair is the major therapeutic option to prevent life-threatening disease progression. Despite increasing knowledge on histopathological features of AAA from human tissue and animal studies, potential targets for prediction of the individual rupture risk and for efficient pharmaceutical interventions to attenuate expansion remain to be identified $(1,2)$.

A driving force in the pathogenesis of AAA is chronic inflammation, resulting in progressive remodeling and deterioration of the aortic wall (3). Recent findings on vascular cell biology have

Address correspondence to Susanne Dihlmann, Department of Vascular and Endovascular Surgery, University of Heidelberg, Im Neuenheimer Feld 110, 69120, Heidelberg, Germany. Phone: + + 49-6221-56-6494; Fax: + + 49-6221-56-7654; E-mail: susanne.dihlmann@med.uni-heidelberg.de. Submitted February 2, 2016; Accepted for Publication July 11, 2016; Published Online (www.molmed.org) July 29, 2016.

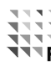

Feinstein Institute
for Medical Research Northwell Health" 
and activation of IL-1 $\beta$ from its inactive precursor form (9). We thus hypothesized that the inflammasome pattern in tissue-infiltrating and peripheral leukocytes of AAA patients might differ from that in individuals without AAA.

The inflammasomes are a family of cytoplasmic multiprotein complexes for danger signal recognition that are induced by pathogens or cell debris (damage associated molecular patterns, (DAMPS)) $(9,10)$. Expression levels of the inflammasome components are tightly regulated in different cell types and require a two-phase induction: a priming signal stimulates gene expression of inactive precursors before a second signal can stimulate assembly of the multiprotein complex consisting of sensor proteins, the adaptor protein apoptosis-associated speck-like protein with a caspase activation and recruitment domain (ASC) and inflammatory caspases (Caspase- 1 or Caspase-5). Upon assembly, the caspases are enzymatically cleaved by the complex resulting in their active form, which then stimulates enzymatic cleavage of IL-1 $\beta$ and Interleukin-18 (IL-18) from inactive precursors (9). Depending on the initial sensor, several subfamilies are distinguished: The NOD-like receptors (NLR), including NLRP3 and others, act as sensors for intracellular damage associated signals such as cholesterol crystals, nanoparticles, reactive oxygen species and others. A second subfamily, including Absent in Melanoma 2 (AIM2) acts as sensors for intracellular dsDNA (9).

The disease-specific regulation of inflammasome expression and activation in circulating blood mononuclear cells of AAA patients is currently unknown. Considering the locally increased level of inflammasomes and inflammasomepriming cytokines in AAA tissue, we investigated whether this pattern is reflected in PBMC of AAA patients. Because it is well accepted that AAA predominantly affects male patients, we additionally analyzed differences in inflammasome activities between male and female AAA and non-AAA individuals.

\section{MATERIALS AND METHODS}

\section{Blood Samples and Patient Characteristics}

Venous blood was taken from vascular patients on the day of their hospitalization, according to the standard operating procedures of the Vascular Biobank Heidelberg (VBBH). All patients gave their written informed consent to the study, which was approved by the ethical committee of the University of Heidelberg (S-301/2013 and S-412/2013). The blood was processed for further studies within $4 \mathrm{~h}$ after venipuncture. A total of 100 blood samples were consecutively included in the study (62 from male, and 38 from female patients) and analyzed for mRNA expression. The reasons for hospitalization (type of vascular disease), additional previous or chronic diseases, as well as sociodemographic and clinical data of the patients were obtained from the hospital's information system. Blood from a subset of 34 AAA patients (hospitalized for open or endovascular intervention of their AAA) was sex- and age-matched to 34 non-AAA patients (hospitalized for treatment of peripheral artery disease (PAD) or carotid artery stenosis, with no evidence for current aortic aneurysms). These 34 pairs were additionally investigated by Western blot and plasma analysis. Patients' characteristics are shown in Table 1. All patients (AAA and controls) were examined for their aortic diameter by duplex sonography, prior
Table 1A. Patient characteristics according to sex.

\begin{tabular}{lccc}
\hline & Male patients (N=62) & Female patients (N=38) & $P$-Value \\
\hline Risk Factors & & & \\
Age (years \pm SD) & $67.50 \pm 9.81$ & $64.18 \pm 12.19$ & 0.138 \\
Smoking (No.) & $61.29 \%(38 / 62)$ & $55.88 \%(19 / 34)$ & 0.667 \\
Hypertension (No.) & $93.44 \%(57 / 61)$ & $91.18 \%(31 / 34)$ & 0.698 \\
Diabetes (No.) & $22.58 \%(14 / 62)$ & $20.59 \%(7 / 34)$ & 1.000 \\
Statin use (No.) & $86.89 \%(53 / 61)$ & $76.47 \%(26 / 34)$ & 0.254 \\
Type of vascular disease & & & \\
AAA (No.) & $43.55 \%(27 / 62)$ & $18.42 \%(7 / 38)$ & 0.016 \\
Carotid stenosis (No.) & $32.26 \%(20 / 62)$ & $21.05 \%(8 / 38)$ & 0.259 \\
PAD (No.) & $16.13 \%(10 / 62)$ & $42.11 \%(16 / 38)$ & 0.005 \\
Other vascular diseases & & & \\
(limb vessel malformations, & $8.06 \%(5 / 62)$ & $18.42 \%(7 / 38)$ & 0.203 \\
angina abdominalis) (No.) & & & \\
\hline
\end{tabular}

Table 1B: Characteristics of patients with AAA and non-AAA (control).

\begin{tabular}{lccc}
\hline & AAA $(\mathrm{N}=34)$ & Control $(\mathrm{N}=34)$ & $P$-Value \\
\hline Risk Factors & & & \\
Age (years) $\pm \mathrm{SD}$ & $68.76 \pm 7.36$ & $69.44 \pm 7.00$ & 0.699 \\
Smoking (No.) & $61.76 \%(21 / 34)$ & $61.76 \%(21 / 34)$ & 1.000 \\
Hypertension (No.) & $97.06 \%(33 / 34)$ & $94.12 \%(32 / 34)$ & 1.000 \\
Diabetes (No.) & $11.76 \%(4 / 34)$ & $35.29 \%(12 / 34)$ & 0.043 \\
Statin use (No.) & $85.29 \%(29 / 34)$ & $79.41 \%(27 / 34)$ & 0.752 \\
Cholesterol level $(\mathrm{mg} / \mathrm{dl}) \pm \mathrm{SD}$ & $175.61 \pm 32.88$ & $164.84 \pm 48.18$ & 0.308 \\
Inflammmatory parameters & & & \\
CRP (mg/dl) $\pm \mathrm{SD}$ & $13.97 \pm 24.61$ & $17.03 \pm 29.89$ & 0.660 \\
Leucocytes $\left(\mathrm{x} 10^{3} / \mathrm{\mu L}\right) \pm \mathrm{SD}$ & $7.79 \pm 1.83$ & $9.08 \pm 3.18$ & 0.049 \\
Max aortic diameter & & & \\
$(\mathrm{cm}) \pm \mathrm{SD}$ & $5.81 \pm 0.95$ & $1.96 \pm 0.57$ & $<0.00001$ \\
\hline
\end{tabular}

aData was analyzed using Student $\dagger$ test or Chi square test. 
to their hospitalization for treatment. All AAA patients and 10 of the control patients were additionally examined by preoperative multislice computed tomographic angiography. For all patients (AAA and controls), the timeframe between their latest aortic imaging and venipuncture for the study was less than six months, generally within one month. Within the control group, three patients had undergone a previous aortic repair due to aneurysm dating back more than five years.

\section{Blood Separation}

Separation of plasma and PBMC from blood was performed as described previously (11). After separation, half of each PBMC pellet was lysed in RIPA buffer $(50 \mathrm{mmol} / \mathrm{L}$ Tris-HCl, pH 7.5, $150 \mathrm{mmol} / \mathrm{L} \mathrm{NaCl}, 0.5 \%$ sodium deoxycholate, $0.1 \%$ SDS, $0.5 \%$ NP-40, complemented with protease inhibitor Mix G, Serva) for protein analysis. The second half was lysed in RLT-buffer (RNeasy mini Kit, Qiagen) for RNA extraction. Lysates were frozen for cryopreservation at $-80^{\circ} \mathrm{C}$ until further usage.

\section{RNA Extraction and qPCR Analysis}

Total RNA was extracted from cryopreserved PBMC pellets using RNeasy mini Kit (Qiagen) according to the manufacturer's instructions. For expression analysis, $0.2 \mu \mathrm{g}$ of the total RNA was reverse transcribed using oligo-dT primers and SuperScript III reverse transcriptase (Life Technologies) as described previously (8). The StepOnePlus Real time PCR System (Life Technologies) was used for mRNA level analysis of AIM2, NLRP3, ASC (PYCARD), CASP1, CASP5 and $I L 1 B$ transcripts. Reagents and qPCR primers have been described previously (11). $C_{T}$ values were analyzed at a $C_{t}$ threshold of 0.34 . Relative mRNA expression of inflammasome genes was calculated by using individual standard curves and normalized to the mean values of three reference genes (GAPDH, B2M, and ACTB).

\section{Western Blotting}

PBMC samples were lysed in RIPA buffer and homogenized by ultrasound. Twenty $\mu \mathrm{g}$ of each lysate were separated by SDS-PAGE an blotted onto nitrocellulose $(0,45 \mu \mathrm{m}$ for detection of AIM2, NLRP3, ASC; 0,2 $\mu \mathrm{m}$ for detection of Caspase-1 (p10 and p50). Paired samples were loaded side by side to ensure comparability. Detection of proteins was performed over night with primary antibodies diluted in appropriate buffers as recommended by the manufacturers. AIM2 (D5X7K) rabbit mAb, NLRP3 (D2P5E) rabbit mAb, cleaved caspase-1 (D57A2) rabbit $\mathrm{mAb}$, and GAPDH (D16H11)XP rabbit mAb were purchased from Cell Signaling Technology, ASC rabbit pAb was obtained from Enzo Life Sciences, Caspase-1 p10 rabbit pAb was from Santa Cruz Biotechnology. Anti-rabbit IgG HRPlinked secondary antibody (Cell Signaling Technology) and Western-lightning plus ECL (Perkin Elmer) were used for detection by chemoluminescence. For relative quantification, signals of equal exposure times were densitometrically analyzed with Image $\mathrm{J}$ and normalized to corresponding GAPDH signals.

\section{ELISA}

Active, cleaved IL-1 $\alpha$ (p18) and IL-1 $\beta$ (p17) in plasma from patients were quantified by the Duo-Set ELISA Development systems for human IL-1 $\alpha$ or IL-1 $\beta$, respectively (R\&D Systems Europe) according to the recommendations of the manufacturer.

\section{Tissue Samples and Immunohistochemistry}

Formalin-fixed and paraffin-embedded biopsies derived from AAA patients were provided by the Vascular Biobank Heidelberg (VBBH) (approved by the ethical committee of the University of Heidelberg; S-412/2013). Processing and immunohistochemical staining was performed following standard procedures as described previously (8) by using the following antibodies: anti-AIM2 (Sigma
Prestige Antibodies; dilution 1:200), anti-NLRP3 (Cell Signaling Technology, dilution 1:500).

\section{Statistical Analysis}

All data was analyzed by IBM SPSS version 21 (IBM Corp). Figures were prepared using GraphPad Prism (GraphPad). Mann Whitney U test was performed to analyze the relative mRNA levels within the 100 vascular patients according to sex. To control for confounding, multivariate analysis was performed by logistic regression for each mRNA. The mRNA levels of male and female patients were adjusted for AAA and PAD, because these variables appeared to differ significantly between both sexes (Table 1A). Next, results from AAA patients were compared with age- and sex-matched controls (vascular patients without AAA) in a matched case-control study. The mRNA and protein levels of matched patients were analyzed by the Wilcoxon signed rank test for univariate analysis. The median, minimum and maximum were determined to be displayed in the figures and tables. To control for confounding, multivariate analysis was performed by conditional logistic regression. Matched pairs of individual mRNA levels were adjusted for history of smoking, hypertension, diabetes and hyperlipidaemia. Spearman's rank correlation coefficients were determined to assess the linear association between mRNA levels and clinical parameters (such as cholesterol level, CRP, leukocyte count, maximal AAA-diameter). $P$ values below 0.05 were interpreted as significant.

All supplementary materials are available online at www.molmed.org.

\section{RESULTS}

\section{Inflammasome-Related mRNA Levels in PBMC Are Significantly Higher in Male than in Female Vascular Patients}

Because women are known to experience lower rates of inflammatory diseases (12) and AAA incidence is 
approximately four times higher in men than in women, we initially compared the relative mRNA levels of AIM2, NLRP3, ASC, CASP1, CASP5 and IL1B in native PBMC of 100 consecutively hospitalized male $(n=62)$ and female $(\mathrm{n}=38)$ patients with different vascular diseases. As shown in Table 2, the relative mRNA levels of all tested inflammasome components were significantly higher in PBMC from male than in PBMC from female patients (AIM2: 1.03 versus $0.73, P=0.00009 ; N L R P 3$ : 1.59 versus $0.58, P=0.000038 ; A S C=$ 2.89 versus $1.24, P=0.000004 ; C A S P 1$ : 1.06 versus $0.67, P=0.000004 ; C A S P 5$ : 1.22 versus $0.18, P<0.000001$; IL1B: 2.34 versus $0.25, P<0.000001)$. In contrast, cardiovascular risk factors such as age, smoking or hypertension did not differ between male and female patients (Table 1A). Moreover, statin use, which might interfere with systemic inflammation, was similar in both sexes (Table 1A).

Univariate logisitc regression revealed highly significant odds ratio (OR) of the individual mRNA levels for male sex (AIM2: $\mathrm{OR}=5.66, P=0.001 ; N L R P 3$ : $\mathrm{OR}=2.04, P=0.005 ; A S C: \mathrm{OR}=1.79$, $P=0.001 ; C A S P 1: \mathrm{OR}=8.75, P=0.0003$; CASP5: $\mathrm{OR}=8.15, P=0.000014 ;$ IL1B: $\mathrm{OR}=1.54, P=0.002)$. After adjustment for AAA and PAD as possible confounding diseases, all inflammasome mRNA levels in PBMC were still significantly associated with male sex (Table 3).

\section{Inflammasome-Related mRNA Levels in PBMC Differ between Male AAA and Non-AAA Control Patients}

As expected, among the consecutively enrolled vascular patients in this study, the rate of AAA patients was significantly higher $(P=0.016)$ in male $(27 / 62)$ than in female $(7 / 38)$ (Table 1A). To further explore inflammasome activities particularly in PBMC of AAA patients, the relative mRNA levels of AIM2, NLRP3, ASC, CASP1, CASP5 and $I L 1 B$ derived from the subgroup of 34 AAA patients were analyzed separately and compared with 34 ageand sex-matched controls. CASP1 and

Table 2. Relative mRNA levels of inflammasome components in PBMC normalized to ACTB/GAPDH/B2M. Data are derived from 62 male and 38 female patients.

\begin{tabular}{lccr}
\hline Target genes & Male Median (range) & Female Median (range) & $P$ - Value \\
\hline AIM2 & $1.03(0.14-9.42)$ & $0.73(0.04-1.72)$ & 0.00009 \\
NLRP3 & $1.59(0.42-9.90)$ & $0.58(0.01-4.88)$ & 0.000038 \\
ASC (PYCARD) & $2.89(0.75-13.74)$ & $1.24(0.39-6.56)$ & 0.000004 \\
CASP1 & $1.06(0.41-4.47)$ & $0.67(0.19-2.25)$ & 0.000004 \\
CASP5 & $1.22(0.00-5.58)$ & $0.18(0.01-2.01)$ & $<0.000001$ \\
IL IB & $2.34(0.07-45.06)$ & $0.25(0.00-10.67)$ & $<0.000001$ \\
\hline
\end{tabular}

aData was analyzed using the Mann Whitney U test.

Table 3. Logistic regression to identify independent variables associated with male sex. Without adjustment With adjustment ${ }^{a}$

\begin{tabular}{|c|c|c|c|c|c|c|}
\hline $\begin{array}{l}\text { Target } \\
\text { Genes }\end{array}$ & $\begin{array}{l}\text { Odds } \\
\text { Ratio }\end{array}$ & $95 \% \mathrm{Cl}$ & P-Value & $\begin{array}{l}\text { Odds } \\
\text { Ratio }\end{array}$ & $95 \% \mathrm{Cl}$ & $P$-Value \\
\hline AIM2 & 5.66 & $2.12-15.09$ & 0.001 & 4.81 & $1.79-12.90$ & 0.002 \\
\hline NLRP3 & 2.04 & $1.24-3.35$ & 0.005 & 1.78 & $1.06-3.01$ & 0.030 \\
\hline ASC & 1.79 & $1.27-2.52$ & 0.001 & 1.71 & $1.20-2.42$ & 0.003 \\
\hline CASP 1 & 8.75 & $2.67-28.68$ & 0.0003 & 7.21 & $2.14-24.29$ & 0.001 \\
\hline CASP5 & 8.15 & $3.16-21.04$ & 0.000014 & 8.66 & $3.03-24.79$ & 0.00006 \\
\hline$I L I B$ & 1.54 & $1.18-2.03$ & 0.002 & 1.51 & $1.13-2.01$ & 0.005 \\
\hline
\end{tabular}

aEach gene was individually adjusted for AAA and PAD.

IL1B mRNA levels were again found to be significantly increased in PBMC of AAA patients (CASP1: $P=0.028$; IL1B: $P=0.0001$, both sexes). In contrast, the relative mRNA levels of AIM2, NLRP3, $A S C$, and CASP5 did not differ between PBMC from AAA patients and controls (Supplementary Figure S1 and Supplementary Table S1).

When male and female AAA patients were analyzed separately, none of the inflammasome mRNAs were found to differ between female AAA and female non-AAA patients (Figure 1 A-F and Supplementary Table S2). In contrast, CASP1 and IL1B mRNA expressions were again found to be significantly increased in PBMC from male AAA patients when compared with matched PBMC from male non-AAA patients (median $_{\mathrm{CASP} 1}=1.21$ versus 1.11, $P=0.032 ;$ median $_{\mathrm{IL} 1 \mathrm{~B}}=4.65$ versus 1.56 , $P=0.0004$, Figure 1D and F). In addition, NLRP3 mRNA was significantly increased in PBMC from male AAA patients (median $_{\mathrm{NLRP} 3}=1.96$ versus1.54; $P=0.044$, Figure 1B and Supplementary Table 2). Finally, male AAA patients showed a higher mRNA expression of AIM2, ASC, CASP1, and CASP5 than female AAA patients in their PBMC (each $P<0.05$; Figure 1).

We next analyzed whether the mRNA levels in male and female AAA patients are associated with clinical parameters (cholesterol level, leukocyte count, CRP, AAA-diameter). No correlations were found between any of the inflammasome mRNA levels and disease parameters in male patients. In contrast, the CASP 5 mRNA level in PBMC of female patients was found to correlate positively $(r=0.775$; $P=0.041)$ with the maximal AAA diameter (Figure 2).

\section{mRNA Levels of Inflammasome Components Correlate with Each Other in PBMC of AAA Patients}

Within the AAA patients, Spearman's correlation test revealed a strong positive correlation of the inflammasome mRNA levels in PBMC with 

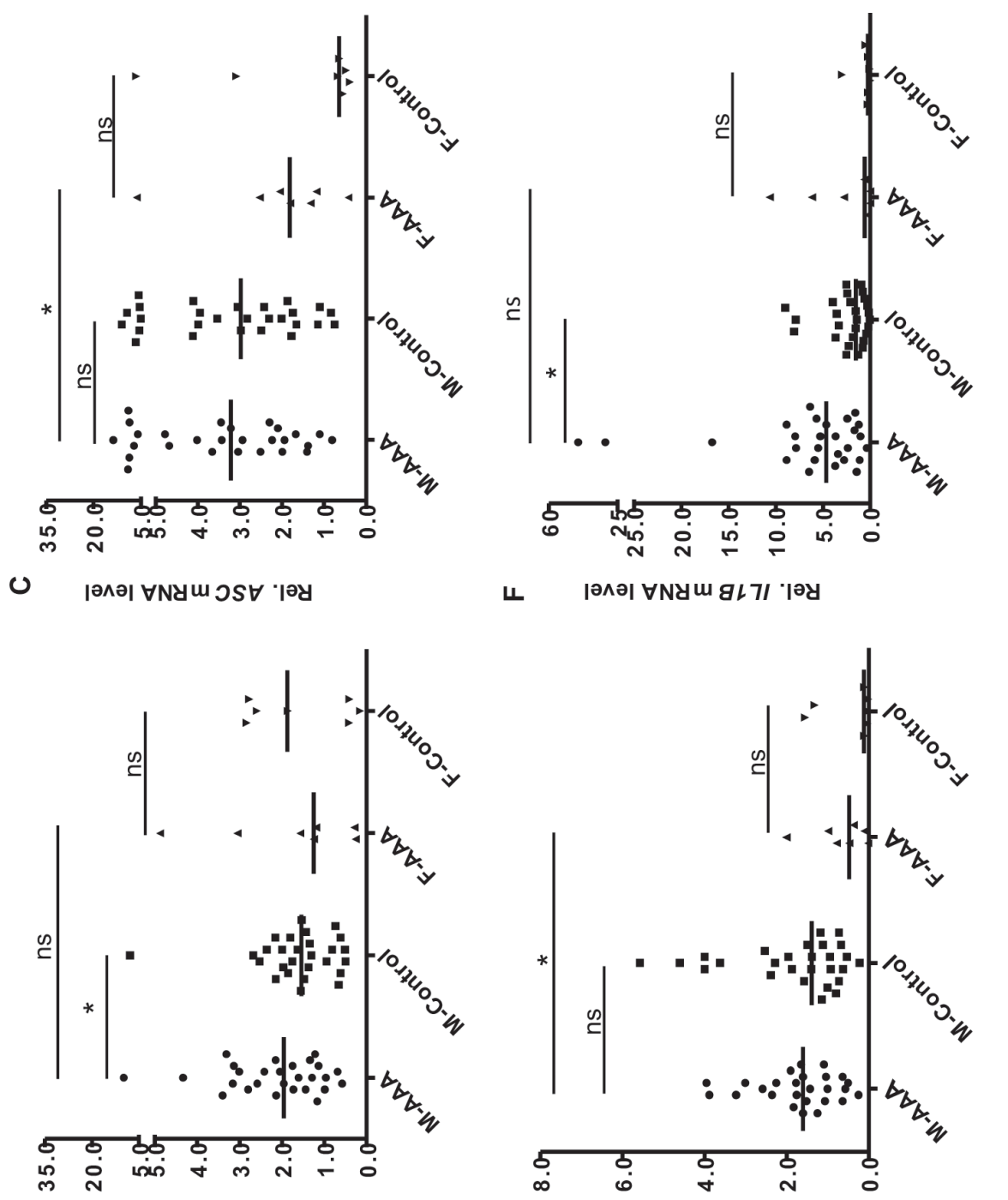

ᄂ

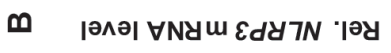
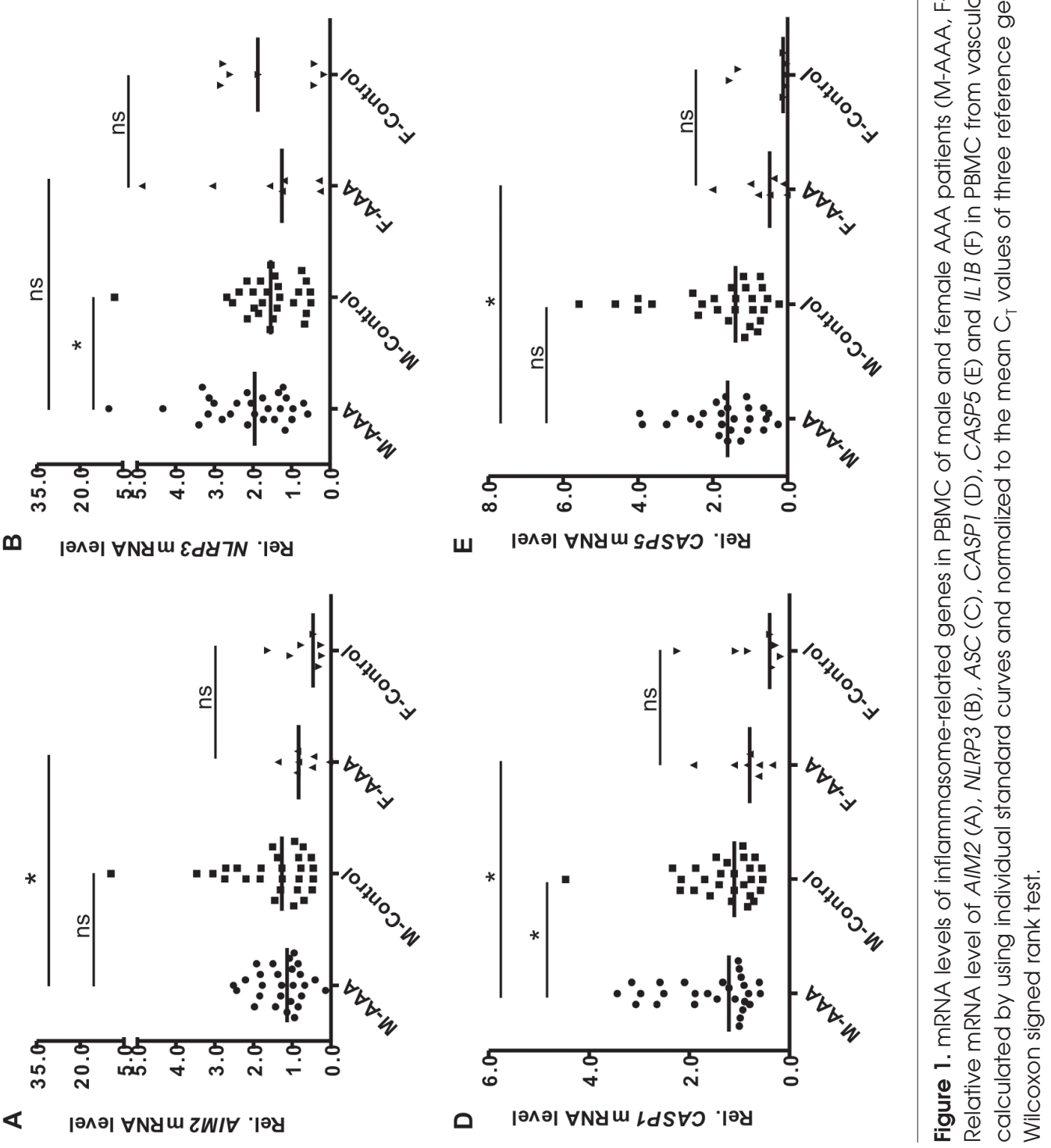


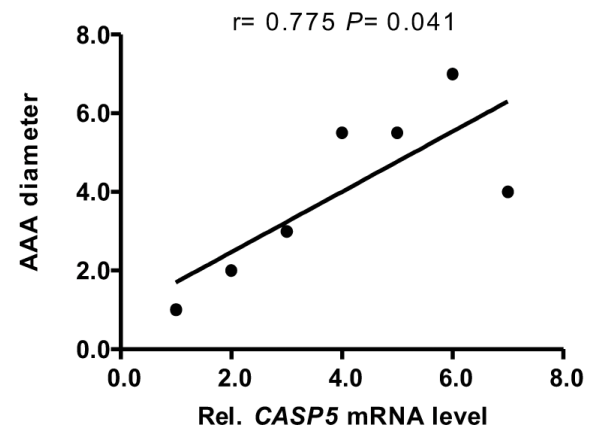

Figure 2. Correlation of CASP5 mRNA expression in PBMC of female AAA-patients. Spearman's rank test was used for analysis.

each other (Supplementary Table S3): The AIM2 mRNA level correlated positively with $A S C(r=0.664 ; P<0.0001)$, CASP1 $(r=0.673 ; P<0.0001)$, CASP5 $(r=0.501 ; P=0.003)$, and IL1B mRNA levels $(r=0.454 ; P=0.007)$. The NLRP3 mRNA level correlated positively with ASC ( $r=0.391 ; P=0.022), C A S P 1$ $(r=0.544 ; P=0.001)$, CASP5 $(r=0.682$; $P<0.0001)$ and $\operatorname{IL1B}(r=0.571$;

$P=0.0004)$. In addition, the ASC mRNA level correlated positively with CASP1 $(r=0.853 ; P<0.0001)$, CASP5 $(r=0.742$; $P<0.0001)$, and IL1B mRNA levels $(r=0.470 ; P=0.005)$. The CASP1 mRNA level correlated positively with CASP5 $(r=0.778 ; P<0.0001)$, and IL1B mRNA levels $(r=0.553 ; P=0.001)$. Finally, the CASP5 mRNA level was positively correlated with IL1B mRNA level $(r=0.578 ; P=0.0004)$. No correlation was found between AIM2 and NLRP3 mRNA levels.

\section{The ILIB mRNA Level in PBMC Is an Independent Risk Factor for AAA}

To test, whether one of the mRNAs is an independent risk factor for AAA, we performed conditional regression analyses. When sex- and age-matched pairs of AAA and control samples were adjusted for history of smoking, hypertension, diabetes and hyperlipidaemia, the IL1B mRNA level in PBMC but not the mRNA levels of other inflammasome components was found to be an independent risk factor for AAA with an (OR) of 2.91 (95\% $\mathrm{CI}=1.01-8.39 ; P=0.048$; Table 4 ).

Table 4. Conditional logistic regression to identify independent risk factors for AAA.

\begin{tabular}{|c|c|c|c|c|c|c|}
\hline \multirow[b]{2}{*}{$\begin{array}{l}\text { Target } \\
\text { Genes }\end{array}$} & \multicolumn{3}{|c|}{ Without adjustment } & \multicolumn{3}{|c|}{ With adjustment ${ }^{a}$} \\
\hline & $\begin{array}{l}\text { Odds } \\
\text { Ratio }\end{array}$ & $95 \% \mathrm{Cl}$ & $P$-Value & $\begin{array}{l}\text { Odds } \\
\text { Ratio }\end{array}$ & $95 \% \mathrm{Cl}$ & P-Value \\
\hline AIM2 & 0.71 & $0.35-1.47$ & 0.358 & 0.99 & $0.43-2.31$ & 0.981 \\
\hline NLRP3 & 1.28 & $0.87-1.90$ & 0.214 & 1.53 & $0.19-12.65$ & 0.693 \\
\hline ASC & 1.14 & $0.90-1.45$ & 0.292 & 0.58 & $0.10-3.25$ & 0.534 \\
\hline CASPI & 2.02 & $0.78-5.24$ & 0.150 & 40.85 & $0.12->999.99$ & 0.213 \\
\hline CASP5 & 0.88 & $0.51-1.50$ & 0.629 & 0.14 & $0.003-5.41$ & 0.289 \\
\hline$I L I B$ & 1.95 & $1.15-3.32$ & 0.014 & 2.91 & $1.01-8.39$ & 0.048 \\
\hline
\end{tabular}

aAdjusted for smoking history, hypertension, diabetes and hyperlipidemia.

\section{AIM2 and Mature Caspase-1 (p10) Protein Levels Indicate Increased Inflammasome Activity in PBMC from AAA Patients}

We next determined protein levels of AIM2, NLRP3, ASC, and Caspase-1 in PBMC lysates by Western blotting. Because lysates were available only from 29 pairs of AAA patients and matched controls (26 male and 3 female), PBMC were not analyzed separately according to sex. In concordance with the mRNA data, the level of ASC protein was similar in PBMC from AAA patients and controls (Figure 3 and Supplementary Table S4). In contrast, the protein levels of the inflammasome sensors AIM2 and NLRP3 did not reflect the corresponding mRNA levels. In fact, the relative AIM2 protein level was significantly higher in PBMC from AAA patients than in other vascular patients (median $=0.98$ versus $0.64 ; P=0.014$; Figure $3 \mathrm{~A}$ ). Interestingly, the damage sensor NLRP3 displayed opposite protein levels. In contrast to the NLRP3 mRNA level, NLRP3 protein levels were significantly lower in PBMC from AAA patients than in other vascular patients (median $=0.03$ versus $0.05 ; P=0.038$; Figure 3B).

Considering that inflammasome assembly and processing of Caspase- 1 (p50) result in cleavage and release of active Caspase-1 (p10), the protein levels of p50 and p10 were analyzed separately. Pro-Caspase-1 (p50) levels were similar in PBMC from all patients (Figure 3D), whereas PBMC from AAA patients contained significantly more active Caspase-1 (p10) than the control group (median $=0.43$ versus $0.34, P=0.049$; Figure 3E).

\section{Mature IL- $1 \alpha$ and IL- $1 \beta$ in Plasma of a Subset of AAA Patients Indicate Inflammasome-Mediated Caspase- 1 Activity}

Circulating mature IL-1 $\alpha$ (p18) and IL-1 $\beta$ (p17) were detected by ELISA in plasma of a subset of patients. Five out of the 34 AAA patients displayed heavily increased IL-1 $\beta$ plasma levels (3.9-178.7 pg/mL). Four out of these five patients simultaneously displayed increased IL-1 $\alpha$ (2.2-103.2 pg/mL). In non-AAA patients, both, mature IL-1 $\alpha$ (p18) and IL-1 $\beta$ (p17) were below $2.0 \mathrm{pg} / \mathrm{mL}$. The median IL-1 $\alpha$ (p18) and IL-1 $\beta$ (p17) concentrations were significantly higher in plasma of AAA-patients compared with controls $(P=0.000018$ and $P=0.00017$, respectively; Figure $3 \mathrm{~F}$ and Supplementary Table S5). Together with the elevated Caspase-1 (p10) levels, this indicates that inflammasome assembly and full functionality is increased in blood cells from AAA patients, in comparison to blood cells from other vascular patients.

\section{AIM2 and NLRP3 Expression in Leukocytic Infiltrates of AAA Tissue \\ Because AIM2 protein levels were increased in PBMC of AAA patients, whereas NLRP3 protein levels were reduced compared with controls, the}



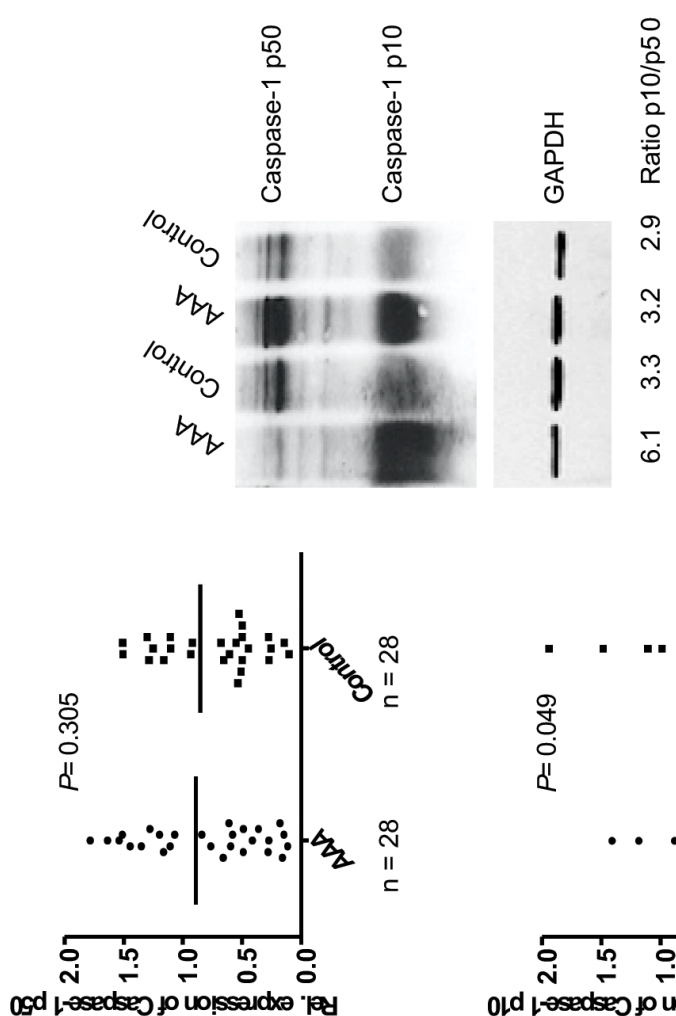

口

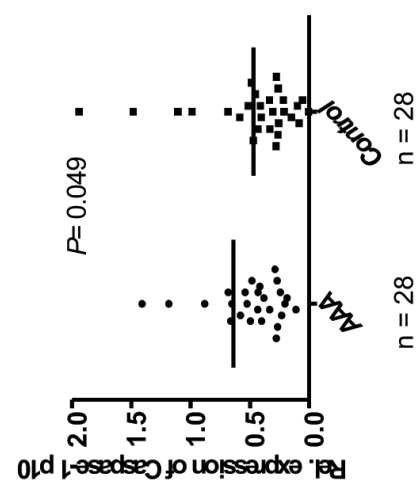

山

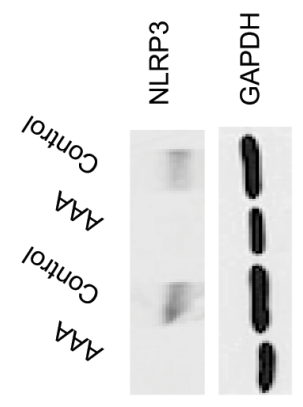

$\varangle$

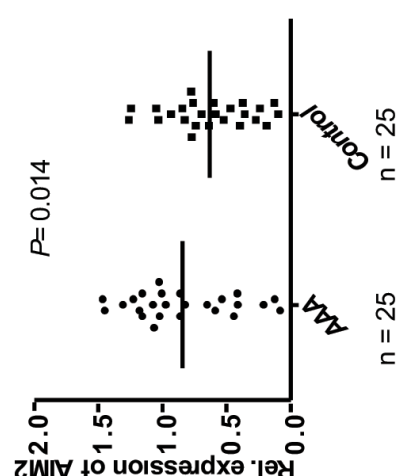

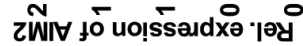

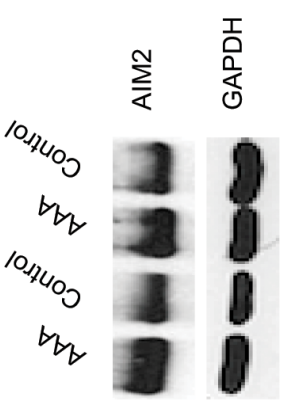

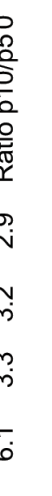

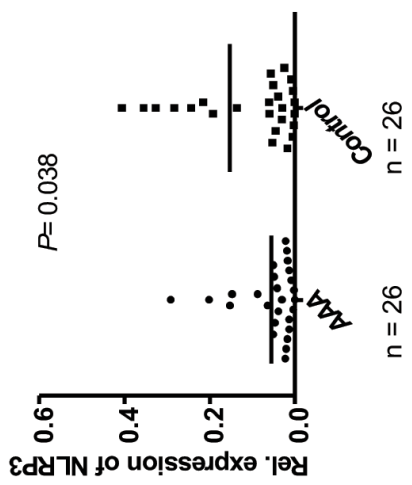

m
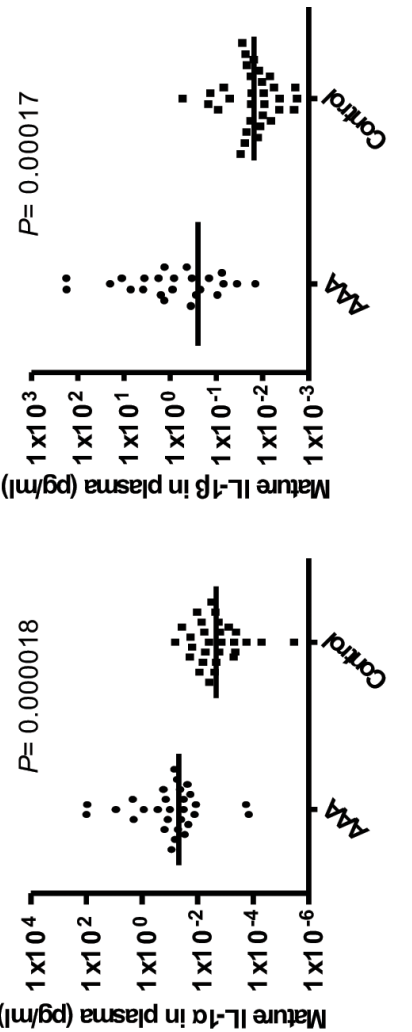

แ

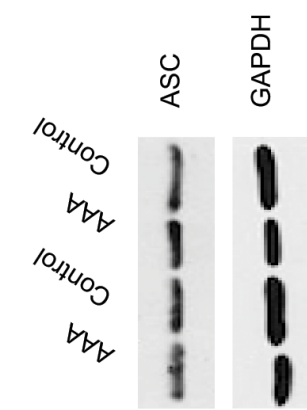

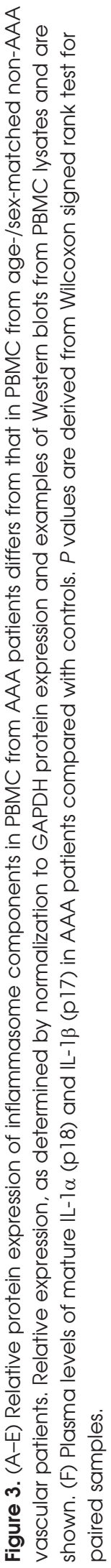


expression of both inflammasome sensors was addressed in corresponding tissue specimens derived from AAA patients. Biopsies of the aneurysm wall corresponding to PBMC samples of the AAA group were available from six male and one female patient, who underwent open surgery for aortic repair. Histological analysis of these biopsies (2-3 tissue specimens from each patient) revealed a high inflammation grade with massive infiltration of leukocytes in the adventitia. In five out of the seven aneurysms, these leukocytic infiltrates displayed strong AIM2 expression, whereas NLRP3 was only detected once by immunohistochemical analysis in adventitia infiltrates. In contrast, AIM2 and NLRP3 positive leukocytes were equally detected in atherosclerotic areas of all aneurysm walls (Figure 4).

To investigate whether this AIM2/ NLRP3 expression pattern is characteristic for AAA, we analyzed 86 additional aneurysm biopsies derived from 42 AAA patients that were provided by the Vascular Biobank Heidelberg (VBBH), by immunohistochemistry. In $97.9 \%$ of AAA patients, AIM2-positive leukocytes were detected in atherosclerotic plaque areas of the intima. Similarly, in $97.9 \%$ of AAA patients, NLRP3-positive leukocytes were detected in these areas of the aneurysm wall. In contrast, the leukocyte infiltrates in the adventitia were more often positive for AIM2 than for NLRP3 expression $(87.0 \%$ versus $58.7 \%$ of patients in grade 2 infiltrates; $50.0 \%$ versus $33.3 \%$ of patients in grade 3 infiltrates) (a more detailed analysis of these biopsies will be published separately).

\section{DISCUSSION}

Differential expression and activation of inflammasomes in PBMC may reflect systemic inflammatory responses via the innate immune system to specific disease states. This study reports that mRNA levels of major inflammasome signaling components are generally higher in PBMC derived from male than female vascular patients, independent from their clinical background and age. Within the group of AAA patients, AIM2, ASC, CASP1, and CASP5 mRNA levels were again found to be significantly higher in PBMC from male than female AAA patients. Furthermore, NLRP3, CASP1 and IL1B mRNA levels were increased in PBMC from male AAA versus male control patients. Together, this indicates a systemic proinflammatory state in male AAA patients with "priming" (transcriptional activation by a first danger signal) of three inflammasome components in their PBMC. In addition, increased protein levels of AIM2 and active Caspase-1 (p10) in PBMC lysates, in combination with elevated IL- $1 \alpha$ and IL-1 $\beta$ levels in corresponding plasma, indicate that AAA patients display increased activities of fully assembled inflammasome complexes in their PBMC.

Sex-dependent differential activation of inflammasome components in immune cells has been reported previously from animal models and patients with other inflammatory diseases: Aim2 mRNA and Aim2 protein levels in splenic cells of male mice have been described to be significantly higher as compared with age-matched female mice, and the male hormone androgen was shown to positively regulate AIM2 expression in immune cells (13). In male patients with systemic lupus erythematosus (SLE), increased AIM2 mRNA was

\section{Patient 1}

\section{Patient 7}
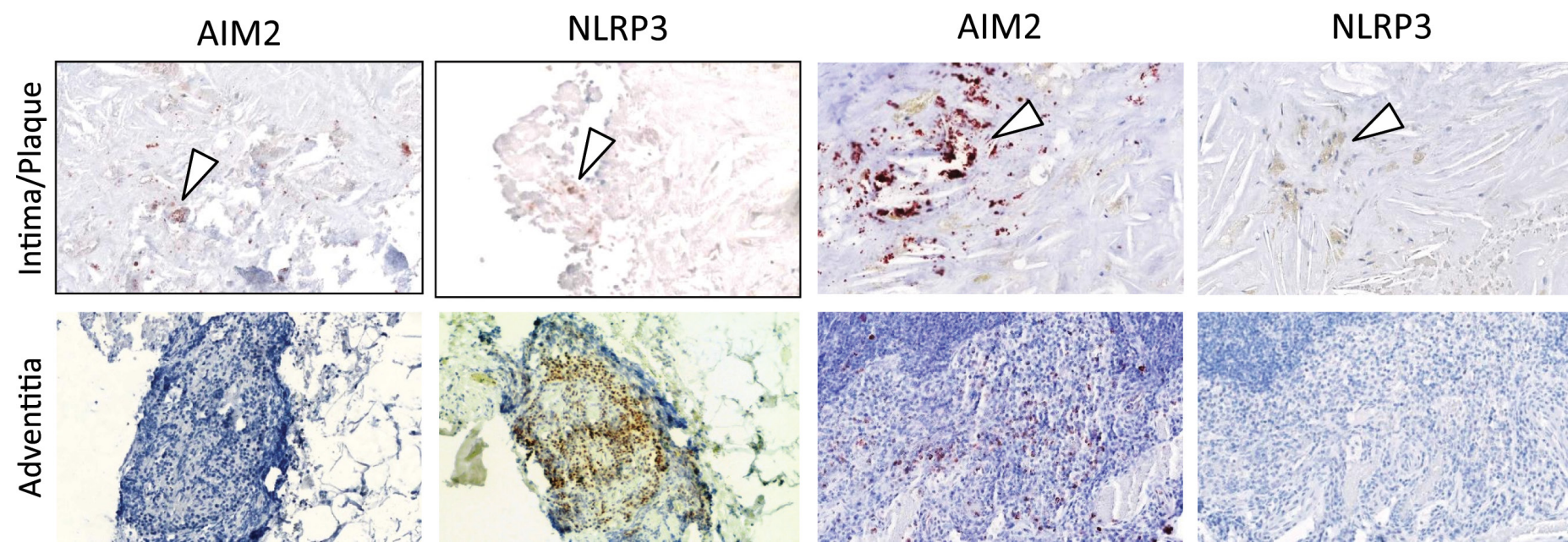

Figure 4. Immunohistochemical analysis of NLRP3 and AIM2 expression in AAA infiltrates. Examples of two AAA biopsies, corresponding to PBMC samples of the same AAA-patient are shown. Arrows point to infiltrating inflammatory/immune cells positive for AIM2 or NLRP3 proteins, respectively. Upper layer: atherosclerotic area at the luminal site. Lower layer: lymphoid infiltrates within the adventitia of the same patient. Original magnifications: 200 -fold. 
detected in unstimulated macrophages, when compared with healthy male controls, whereas NLRP3 mRNA was found to be elevated in female SLE patients as compared with female healthy individuals. (14). Our study adds to these findings by demonstrating that in vascular patients, mRNA levels of the inflammasome components AIM2, NLRP3, ASC, CASP1, CASP5 and IL1B in PBMC are basically higher in male than in female individuals. Furthermore, NLRP3, CASP1 and IL1B mRNA levels appear to be increased in PBMC from male AAA versus male control patients, whereas no difference was found between female AAA and non-AAA patients. On the one hand, this sex difference in inflammasome priming may explain in part why male sex is a risk factor for AAA development. On the other hand, these variations illustrate the importance of analyzing mRNA levels in peripheral blood cells separately according to sex and disease. Obviously, the patient's sex strongly impacts the innate immune response to certain inflammatory diseases. In addition, we have recently reported that mRNA levels of inflammasome components in PBMC increase with age (11). Consequently, the AAA patients of the present study were matched for both sex and age, to detect true AAA-related differences of inflammasome activities in PBMC.

Increased mRNA levels of inflammasome components in PBMC have also been reported from other cardiovascular diseases. Similar to our findings in AAA patients, the mRNA levels of NLRP3, CASP1 and IL1B were found to be increased in peripheral blood monocytes from patients with coronary artery disease / atherosclerosis compared with controls (patients with stable angina or acute coronary syndrome, respectively) (15). Thus, the simultaneously increased mRNA levels of NLRP3, CASP1 and IL1B appear to be associated with a systemic proinflammatory state of peripheral blood monocytes in the background of an advanced atherosclerotic disease such as coronary heart disease or AAA.
In contrast, NLRP3, CASP1 and IL1B mRNA levels were found to be decreased in PBMC from patients with autoimmune diseases, such as SLE patients (16) or patients with rheumatoid arthritis, as compared with age- and sex-matched healthy individuals (17). PBMC are a mix of different peripheral immune cells, composed of monocytes, B- and T-lymphocytes. The coordinated regulation of three inflammasome genes suggests that it may stem from one certain cell type. This hypothesis is further supported by our observation that the levels of all inflammasome mRNAs in PBMC from AAA and non-AAA patients strongly correlated with each other. However, it is unclear so far whether the inflammasome genes are transcriptionally upregulated in one cell type, that is, monocytes, or whether the percentage of this cell type-expressing constant levels of inflammasome genes per cellis increased within the PBMC of AAA patients.

Of note, one inflammasome component was closely associated with disease parameters in AAA: CASP5 mRNA levels were positively correlated with the maximal diameter of the aneurysm in female, but not in male AAA-patients. Moreover, median CASP5 mRNA levels were higher in female AAA-patients compared with female non-AAA patients, although the difference was not significant. This data is based on the limited number of seven female AAA-patients, so far, and needs further investigation within a larger patient cohort before it may be considered as a potential peripheral blood biomarker for AAA progression in female.

To our knowledge, this is the first study showing that the IL1B mRNA level in PBMC is an independent risk factor for AAA. The data corresponds well with previous studies on AAA tissue, where IL1B mRNA levels were found to be increased compared with healthy aortas $(7,8)$. IL1B encodes the inactive precursor of IL- $1 \beta$ that requires Caspase-1 mediated enzymatic cleavage to become active. Increase of IL1B may thus be considered an initiating, ratelimiting step in the priming of inflammasome function (9). Consequently, the increased IL1B mRNA level in PBMC of AAA patients could indicate early steps of an inflammatory response to the tissue remodeling in the aortic wall.

Besides detecting increased inflammasome mRNA levels, our study provides evidence for full inflammasome assembly and increased Caspase-1 activation in PBMC from AAA-patients. Both active Caspase-1 (p10) and its cleavage products, mature IL-1 $\alpha$ (p18) and IL-1 $\beta$ (p17), were elevated in peripheral blood of AAA-patients, demonstrating a strong inflammatory response. Our data are in line with previous reports on IL-1 plasma/serum levels of AAA patients. Yates et al., have shown increased IL-1 $\alpha$ serum levels in AAA-patients that correlated positively with the maximal AAA-diameter before surgery (18). Interestingly, IL-1 $\alpha$ serum levels dropped significantly six months after endovascular repair in these patients. Significantly higher amounts of circulating IL-1 $\beta$ were found to be associated with AAA in a study of 50 AAA patients, 42 patients with coronary heart disease and 38 healthy controls (19). The importance of circulating IL-1 $\beta$ in AAA pathogenesis is further emphasized by animal studies, demonstrating that genetic and pharmacologic disruption of IL-1 $\beta$ signaling inhibits experimental abdominal and thoracic aortic aneurysms formation $(20,21)$. Finally, since it is well accepted that blood monocytes from patients with autoinflammatory syndromes release more processed IL-1 $\beta$ than cells from healthy subjects and thus likely account for the inflammation in these diseases $(22,23)$, AAA may be considered an autoinflammatory disease.

It should be noted here that maturation and release of IL- $1 \alpha$ and IL- $1 \beta$ is not fully restricted to inflammasome-mediated caspase- 1 activity $(24,25)$ and that caspase- 1 appears to have additional functions independent of IL-1 maturation (26). Thus, although our data suggest a role of inflammasome-mediated 
activation of caspase-1 and IL-1 in PBMC of AAA patients, the precise cellular origin of these compounds and their activation mechanism in AAA needs to be investigated. Future analysis of peripheral blood cells by flow cytometry, which is currently performed in our research group, will help to differentiate inflammasome regulation in different peripheral immunophenotypes from patients with AAA and thus elucidate their contribution to IL-1 production and pathogenesis. In addition to inflammasome activities in peripheral blood leukocytes, infiltrating inflammatory/immune cells within the aneurysm wall may likewise contribute to IL-1 concentrations in plasma.

Interestingly, here AIM2 protein levels were increased, whereas NLRP3 protein levels were decreased in PBMC of AAA patients. This indicates a predominant role of AIM2 inflammasomes in AAA. The immunohistochemical analysis of corresponding AAA-tissue from patients supported this hypothesis: In five out of seven corresponding aneurysms, leukocytic infiltrates displayed strong AIM2 protein expression, whereas NLRP3 was only detected once by immunohistochemical analysis in adventitia infiltrates. In additional 86 biopsies of AAA adventitia, AIM2 protein expression was again much more prominent than NLRP3 protein expression. Whether these adventitia-infiltrating immune cells are derived from peripheral blood cells that invaded the tissue, and whether inflammasome activation occurred before or after tissue infiltration remain to be investigated. There is clear evidence that cell death can activate innate immunity via inflammasomes and result in sterile imflammation also in the vasculature (27). Possibly, necrotic cells from the aneurysm wall release intracellular material, which triggers inflammasome activation via the dsDNA sensor AIM2 and invasion of immune cells from the peripheral blood.

\section{CONCLUSION}

In summary, our study provides evidence for a specific (sex-dependent) inflammasome signature in PBMC of
AAA-patients, and suggests an active role of the AIM2 inflammasome in both peripheral blood and adventitia-infiltrating leukocytes in AAA pathogenesis. Particularly, the IL1B mRNA level in PBMC that was found to be an independent risk factor for AAA, warrants further investigation as a putative biomarker for disease progression and rupture risk in AAA patients, as well as a therapeutic target.

\section{ACKNOWLEDGMENTS}

We thank Anja Spieler for excellent technical assistance, Sven Schnaidt (Medical Biometry and Informatics, University Hospital Heidelberg) for statistical counseling and Lei Huang (Dept. of Clinical Epidemiology and Ageing research, German Cancer Research center, DKFZ, Heidelberg) for assistance with the conditional logistic regression analysis.

\section{DISCLOSURE}

The study was funded by an Oversea Study Fellowship from the China Scholarship Council to Xiaoyu Wu. We acknowledge financial support by the Department of Vascular and Endovascular surgery of the University of Heidelberg, Germany. In addition, we acknowledge financial support by Deutsche Forschungsgemeinschaft and Ruprecht-Karls-Universität Heidelberg within the funding program Open Access Publishing.

\section{REFERENCES}

1. Bergqvist D. (2011) Pharmacological interventions to attenuate the expansion of abdominal aortic aneurysm (AAA) - a systematic review. Eur. J. Vasc. Endovasc. Surg. 41:663-667.

2. Dale MA, Ruhlman MK, Baxter BT. (2015) Inflammatory cell phenotypes in AAAs: their role and potential as targets for therapy. Arterioscler. Thromb. Vasc. Biol. 35:1746-1755.

3. Shimizu K, Mitchell RN, Libby P. (2006) Inflammation and cellular immune responses in abdominal aortic aneurysms. Arterioscler. Thromb Vasc. Biol. 26:987-994.

4. Rizas KD, Ippagunta N, Tilson MD, 3rd. (2009) Immune cells and molecular mediators in the pathogenesis of the abdominal aortic aneurysm. Cardiol. Rev. 17:201-210.

5. Tsuruda T, et al. (2008) Adventitial mast cells contribute to pathogenesis in the progression of abdominal aortic aneurysm. Circ. Res. 102:1368-1377.
6. Cohen JR, et al. (1991) Neutrophil chemotaxis and neutrophil elastase in the aortic wall in patients with abdominal aortic aneurysms. J. Invest. Surg. 4:423-430.

7. Golledge AL, Walker P, Norman PE, Golledge J. (2009) A systematic review of studies examining inflammation associated cytokines in human abdominal aortic aneurysm samples. Dis. Markers. 26:181-188.

8. Dihlmann S, et al. (2014) Increased expression and activation of absent in melanoma 2 inflammasome components in lymphocytic infiltrates of abdominal aortic aneurysms. Mol. Med. 19;20:230-237.

9. Latz E, Xiao TS, Stutz A. (2013) Activation and regulation of the inflammasomes. Nat. Rev. Immunol. 13:397-411.

10. Kono H, Kimura Y, Latz E. (2014) Inflammasome activation in response to dead cells and their metabolites. Curr. Opin. Immunol. 30:91-98.

11. Wu X, et al. (2015) Gene expression of inflammasome components in peripheral blood mononuclear cells (PBMC) of vascular patients increases with age. Immun. Ageing. 12:15.

12. Gubbels Bupp MR. (2015) Sex, the aging immune system, and chronic disease. Cell. Immunol. 294:102-110.

13. Panchanathan R, et al. (2011) Cell type and gender-dependent differential regulation of the p202 and Aim2 proteins: implications for the regulation of innate immune responses in SLE. Mol. Immunol. 49(1-2):273-280.

14. Yang CA, Huang ST, Chiang BL. (2015) Sexdependent differential activation of NLRP3 and AIM2 inflammasomes in SLE macrophages. Rheumatology. 54:324-331.

15. Wang L, Qu P, Zhao J, Chang Y. (2014) NLRP3 and downstream cytokine expression elevated in the monocytes of patients with coronary artery disease. Arch. Med. Sci. 10:791-800.

16. Yang Q, et al. (2014) Deregulated NLRP3 and NLRP1 inflammasomes and their correlations with disease activity in systemic lupus erythematosus. J. Rheumatol. 41:444-452.

17. Wang T, et al. (2014) Role of NLRP3 and NLRP1 inflammasomes signaling pathways in pathogenesis of rheumatoid arthritis. Asian Pac. J. Trop. Med. 7:827-831.

18. Yates CM, et al. (2011) Endovascular aneurysm repair reverses the increased titer and the inflammatory activity of interleukin-1alpha in the serum of patients with abdominal aortic aneurysm. J. Vasc. Surg. 54:497-503.

19. Juvonen J, et al. (1997) Elevated circulating levels of inflammatory cytokines in patients with abdominal aortic aneurysm. Arterioscler. Thromb. Vasc. Biol. 17:2843-2847.

20. Johnston WF, et al. (2013) Genetic and pharmacologic disruption of interleukin-1beta signaling inhibits experimental aortic aneurysm formation. Arterioscler. Thromb. Vasc. Biol. 33:294-304.

21. Johnston WF, et al. (2014) Inhibition of interleukin-1beta decreases aneurysm formation and 
progression in a novel model of thoracic aortic aneurysms. Circulation. 130(11 Suppl 1):S51-S59.

22. Dinarello CA. (2011) A clinical perspective of

IL-1beta as the gatekeeper of inflammation. Eur. J. Immunol. 41:1203-1217.

23. Dinarello CA. (2011) Interleukin-1 in the pathogenesis and treatment of inflammatory diseases. Blood. 117:3720-3732.

24. Gross $\mathrm{O}$, et al. (2012) Inflammasome activators induce interleukin-1alpha secretion via distinct pathways with differential requirement for the protease function of caspase-1. Immunity. 36:388-400.

25. Monie TP, Bryant CE. (2015) Caspase-8 functions as a key mediator of inflammation and proIL-1beta processing via both canonical and noncanonical pathways. Immunol. Rev. 265:181-193.

26. Sollberger G, Strittmatter GE, Garstkiewicz M, Sand J, Beer HD. (2014) Caspase-1: the inflammasome and beyond. Innate Immun. 20:115-125.

27. Zheng Y, Gardner SE, Clarke MC. (2011) Cell death, damage-associated molecular patterns, and sterile inflammation in cardiovascular disease. Arterioscler. Thromb. Vasc. Biol. 31:2781-2786.

Cite this article as: Wu X, et al. (2016) Sex- and disease-specific inflammasome signatures in circulating blood leukocytes of patients with abdominal aortic aneurysm. Mol. Med. 22:508-18. 